\title{
Renal Function Variability: An Independent Risk Factor for Graft Loss and Death following Kidney Transplantation
}

\author{
Nicole A. Pilch ${ }^{a}$ Vinayak Rohan ${ }^{b}$ Vinaya Rao ${ }^{c}$ Patrick D. Mauldin ${ }^{d}$ Zemin Su ${ }^{d}$ \\ Derek A. Dubay ${ }^{b}$ Thomas A. Morinellib David J. Taber ${ }^{b}$ e \\ ${ }^{a}$ Department of Clinical Pharmacy and Outcomes Sciences, College of Pharmacy, Medical University of South Carolina, \\ Charleston, SC, USA; ${ }^{b}$ Department of Surgery, Medical University of South Carolina, Charleston, SC, USA; ${ }^{\circ}$ Department \\ of Medicine, Medical University of South Carolina, Charleston, SC, USA; ${ }^{\mathrm{d}}$ Division of General Internal Medicine and

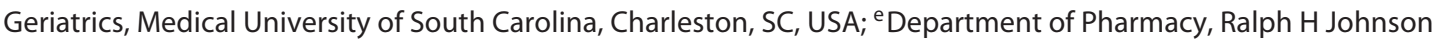 \\ VAMC, Charleston, SC, USA
}

\section{Keywords}

Kidney · Transplantation · Glomerular filtration rate $\cdot$ Risk factors

\begin{abstract}
Background: Several studies have been performed to evaluate surrogate markers of long-term allograft function in renal transplant recipients. These include serum creatinine, estimated glomerular filtration rate (eGFR), slope of eGFR, and more recently eGFR variability. The aim of this study was to measure eGFR slope while assessing the variability of this slope and if high variability occurring at any time post-transplant was predictive of poorer long-term outcomes in a large cohort of kidney transplant recipients. Methods: Adult solitary kidney transplant recipients transplanted between July 1, 2005 and July 31, 2015 were included. The primary outcome was time to graft loss, defined as return to chronic dialysis, retransplant, or death. Second-
\end{abstract}

ary outcomes were death-censored graft loss and acute allograft rejection. Cox regression was utilized for primary and secondary outcomes. Multivariate logistic regression was used to determine baseline factors predictive of high eGFR variability. Results: A total of 1,543 patients were included in the analysis. The percentage of patients who experienced an eGFR coefficient of variation of $<30 \%$ was $79.6 \%(1,229 / 1,543)$, while $20.4 \%(314 / 1,543)$ patients had high eGFR variability ( $\geq 30 \%$ ). Patients with high eGFR variability tended to be younger, African-American and female. Those with higher eGFR variability, accounting for confounding and other eGFR measures (peak and slope), had significantly lower overall patient and graft survival. Conclusion: This study provides a novel analysis of the utility of eGFR variability in a large cohort. The clinical use of the slope of eGFR and eGFR variability may aid in predicting long-term graft outcomes and facilitate early patient discussions to change the trajectory of allograft function.

(c) 2018 S. Karger AG, Basel

\section{KARGER}

(c) 2018 S. Karger AG, Basel

E-Mail karger@karger.com

www.karger.com/ajn
Nicole A. Pilch, PharmD, MSCR, BCPS

Director Quality and Outcomes Transplant ICCE, Department of Clinical Pharmacy and Outcomes Sciences, College of Pharmacy, Medical University of South Carolina 280 Calhoun Street, MSC140, Charleston, SC 29425 (USA)

E-Mail weimert@musc.edu 


\section{Introduction}

Despite advances in immunosuppression and immune monitoring, long-term kidney allograft survival has not substantially improved in recent years [1-3]. Tenyear allograft failure for deceased donor transplant recipients was 52.8\% in 2005 versus 59.2\% in 1995 [3]. As expected, graft failure is the highest in those patients with diabetes and hypertension as the etiology of their endstage renal disease [3]. Currently, only limited data are available to predict progression to chronic kidney disease in patients who receive a kidney transplant. The recent evolution in recipient demographics and expansion of the donor pool have introduced several important variables that may contribute to the lack of improvement in longterm allograft survival but may be better matched with predicted patient survival [4-7]. Although the changes in the kidney allocation scoring system have led to presumed improved equity in organ availability for disadvantaged recipients, the transplant community is in need of an objective measure to aid in determining which patients are at the highest risk for graft failure [5-8]. Implementation of an easy, cost-effective measure that can be utilized during routine lab review to target patients for intervention is needed.

Lenihan et al. [9] evaluated 1-year estimated glomerular filtration rate (eGFR) utilizing the modification of diet in renal disease equation and serum creatinine as predictors of long-term outcomes in patients transplanted between 1989 and 2005. Their cohort included 1,110 patients whose graft was still functioning at 1 year and evaluated them for 10 years. Cox regression of graft outcomes indicated that 1-year eGFR had the highest hazard ratio (HR) at 1.701 (95\% CI 1.45-2.01) in death-censored variables among traditional variables thought to predict graft loss, including acute rejection (HR 1.442, 95\% CI 1.0611.962), repeat transplant (HR 1.345, 95\% CI 0.935-1.936), or presence of delayed graft function following transplant (HR 0.938, 95\% CI 0.556-1.584) [9]. Authors identified a cut point of $<27 \mathrm{~mL} / \mathrm{min} / 1.73 \mathrm{~m}^{2}$ as a threshold value for predicting rapid graft loss [9]. These results were echoed in a more recent trial by Lam et al. [10] who evaluated eGFR and albuminuria in kidney transplant recipients who experienced allograft loss. The investigators evaluated 900 kidney transplant recipients and found that death-censored graft loss correlated significantly with reduced eGFR, and when combined with albuminuria, the association was even more profound [10].

Although eGFR at 1 year is predictive of long-term outcomes, it does not provide insight into what is oc- curring leading up to the 1 year or the trajectory of graft function following 1-year post-transplant. The slope of eGFR has been studied in kidney transplant recipients early post-transplant to predict the recovery of function [11]. More importantly, the slope of estimated GFR has also been studied as a predictor of long-term allograft and patient outcomes. The slope of eGFR allows clinicians to evaluate a more complete picture of the patient's postoperative course. Instead of looking at the last couple of serum creatinine or eGFR values during a post-transplant encounter, eGFR slope demonstrates what has been happening over a long period of time. $\mathrm{Wu}$ et al. [12] performed a retrospective analysis of deceased donor recipients who underwent transplant between 1992 and 2003. Utilizing a slope generated from eGFR calculations from serum creatinine values obtained at month $1,2,3,6,12$, and annually thereafter, authors were able to demonstrate that a slope of $<-2 \mathrm{~mL} / \mathrm{min} / \mathrm{month}$ was predictive of poor long-term allograft outcomes (HR 1.683 with 95\% CI 1.12-2.527) in univariate analysis, specifically when combined with 1 year GFR $<45 \mathrm{~mL} / \mathrm{min}$ [12]. Similar findings were also reported by Park et al. [13] where they looked in change in eGFR between 3 and 12 months post-transplant and divided their cohort into 3 groups $(\geq 10 \%$, between -10 and $10 \%,<-10 \%)$ to evaluate long-term outcomes. The slope of eGFR provides additional insight into eGFR trajectory; however, in evaluation of our data it did not provide a precision required to identify patients for intervention.

Interestingly, Choi et al. [14] recently published a retrospective cohort study on patients transplanted between 1979 and 2012 to determine if eGFR variability in the first year was predictive of patient and allograft survival. This primarily living donor ( $88.4 \%$ living donor vs. $11.6 \%$ deceased donor) study excluded patients with poor early graft function (eGFR $<30 \mathrm{~mL} / \mathrm{min} / \mathrm{m}^{2}$ ) at 1 month and those patients without follow-up eGFR measurements. This study divided patients into 4 quartile ranges based on eGFR-coefficient of variation (CV) cutoff of 0.1832 for those without acute rejection. Sub-analysis was performed on patients with and without acute rejection in the first year. Authors found that high eGFR variability in the first year after kidney transplant was independently associated with graft failure and with all-cause mortality in those without acute rejection [14]. This study had a number of limitations. First, eGFR variability was assessed by using only 4 eGFR measurements (every 3 months for the first year after transplant). Second, the cohort contained patients transplanted over a 30 -year pe- 
riod and the patients are not representative of contemporary kidney recipients with modern immunosuppression. Third, the multivariable models were not adjusted for all potential confounders and did not assess other eGFR variables. For these reasons, further research into eGFR variability and its impact on outcomes is needed.

We postulated that eGFR at 1 year and eGFR variability at 1 year, although are good predictors of long-term outcomes, the short duration of these metrics did not capture patients with significant events, which alter the slope of eGFR overtime and could be targeted for intervention. Therefore, the aim of this study was to measure eGFR slope while assessing the variability of this slope and if high variability occurring at any time post transplant was predictive of poorer long-term outcomes.

\section{Materials and Methods}

\section{Study Design and Patients}

We conducted a cohort study in kidney transplant recipients by using comprehensive baseline and follow-up information acquired retrospectively by querying, cleaning, and analyzing data available from the electronic health record, which we linked to center-specific data (Standard Transplant Analysis Files) supplied by the United Network of Organ Sharing. Kidney transplant recipients were included in this study if they received a transplant between July 1, 2005 and July 31, 2015. Patients were followed through July 31,2015 to allow for $\geq 1$ month of follow-up. Those $<18$ years of age at the time of transplant, those who received non-renal transplants and those without eGFR data sufficient to assess variability were excluded from the study (online suppl. Fig. 2; for all online suppl. material, see www.karger.com/ doi/10.1159/000487714). We received local IRB-expedited approval to conduct this study.

\section{Primary Exposure and Covariates}

The primary exposure variable of interest was eGFR variability (eGFR, $\mathrm{mL} / \mathrm{min} / 1.73 \mathrm{~m}^{2}$ ), which was defined as the intra-patient mean $\mathrm{CV}\left(\% \mathrm{CV}=[\sigma / \mu]^{*} 100\right)$ assessed during the follow-up period as defined below. The eGFR was calculated using the 4-variable modification of diet in renal disease [15]. To determine eGFR variability, we first identified the peak eGFR that occurred in each patient during the first-year post transplant. From this time point, all subsequent eGFR measurements were utilized to determine eGFR variability, until such time as the patient developed graft loss, died or reached the end of follow-up. Additional eGFR variables that were estimated and included in analyses were first-year peak, slope after first-year peak, and mean after first-year peak, which were estimated in a similar manner to the eGFR variability. Online supplemental Figure 1 provides a visual example of a study patient's posttransplant eGFR measures and how each variable was estimated.

Beyond eGFR measures, additional variables assessed for this analysis included recipient demographics (age, sex, race, weight, comorbidities, previous transplant, time on dialysis), donor information (age, sex, race, living, deceased,), transplant characteristics (panel reactive antibody, human leukocyte antigen mismatches, cold ischemic time, need for dialysis within 7 days post-transplant), and immunosuppression (induction and maintenance therapy and tacrolimus trough concentrations).

\section{Outcomes}

The primary outcome for this study was time to graft loss, defined as a return to chronic dialysis, retransplantation, or death. Time to death was assessed as a secondary clinical outcome, as was death-censored graft loss and acute allograft rejection. Rejection was defined as biopsy proven and treated, based on Banff '97 criteria (severity $\geq 1 \mathrm{~A}$ ) or evidence of antibody-mediated rejection.

\section{Statistical Analysis}

The summary descriptive statistics was utilized based on the data type - means \pm SD for continuous normally distributed data, medians with interquartile ranges for ordinal and non-normally distributed continuous data and percentages for categorical data. Two-sided univariate comparisons were conducted using the independent $t$ test, Mann-Whitney $\mathrm{U}$ test, or chi-square test as appropriate, based on data type and distribution. Cox regression was utilized for the primary and secondary outcomes, using time-toevent analyses. Multivariable logistic regression was used to determine which baseline factors were predictive of high eGFR variability $(\mathrm{CV} \geq 30 \%)$. The backwards selection process with an exit $p$ value at the $10 \%$ level was used for variable selection. Correlations among independent variables were assessed for multicollinearity. Statistical significance was determined at the 5\% level. SPSS version 23.0 was utilized for this study.

\section{Results}

A total of 1,925 patients were transplanted between July 2005 and July 2015; 1,543 KTX recipients were included in the final cohort after excluding pediatric, non-renal transplant recipients and those without eGFR data (online suppl. Fig. 2). A total of 1,229 patients had an eGFR CV of $<30 \%$ and $314(20.4 \%)$ developed high eGFR variability after transplant $(\mathrm{CV} \geq 30 \%)$. Baseline transplant and recipient characteristics are presented in Table 1 . The patients in the group with high eGFR variability tended to be younger, African-American, females and had hypertension as the primary diagnosis for renal failure. This group also tended to have older female donors. Patients in the eGFR CV $<30 \%$ received IL2RA induction therapy more often and were less likely to be on an mammalian target of rapamycin inhibitor.

\section{Post-Transplant Outcomes}

Post-transplant outcomes are detailed in Table 2. Patients with eGFR CV $\geq 30 \%$ had significantly higher incidence of biopsy-proven acute rejection, despite receiving more cytolytic induction therapy and having similar tacrolimus trough concentrations ( 32.5 vs. $8.9 \%, p<0.001$ ). 
Table 1. Baseline recipient demographics, donor information, and transplant characteristics stratified and compared eGFR variability

\begin{tabular}{|c|c|c|c|}
\hline Characteristic & $\begin{array}{l}\text { eGFR CV <30\% } \\
(n=1,229)\end{array}$ & $\begin{array}{l}\text { eGFR CV } \geq 30 \% \\
(n=314)\end{array}$ & $p$ value \\
\hline Age, years, mean \pm SD & $51.8 \pm 13.5$ & $49.8 \pm 14.9$ & 0.029 \\
\hline Gender, female, $\%$ & 37.7 & 44.9 & 0.019 \\
\hline African-American, $\%$ & 52.6 & 58.9 & 0.047 \\
\hline Weight, kg, mean \pm SD & $84.8 \pm 19.0$ & $84.4 \pm 19.2$ & 0.783 \\
\hline \multicolumn{4}{|l|}{ Primary diagnosis for ESRD, \% } \\
\hline Hypertension & 29.1 & 36.9 & 0.007 \\
\hline Diabetes & 26.0 & 22.0 & 0.147 \\
\hline Glomerular disease & 15.2 & 16.9 & 0.468 \\
\hline History of diabetes, $\%$ & 33.1 & 34.7 & 0.592 \\
\hline Dialysis prior to transplant, $\%$ & 81.6 & 84.4 & 0.250 \\
\hline Time on dialysis, years, mean \pm SD & $2.9 \pm 2.8$ & $3.0 \pm 2.7$ & 0.340 \\
\hline Previous kidney transplant, $\%$ & 7.6 & 5.7 & 0.261 \\
\hline Donor age, years, mean \pm SD & $36.3 \pm 15.5$ & $39.3 \pm 15.3$ & 0.003 \\
\hline Donor gender, female, $\%$ & 40.8 & 49.0 & 0.009 \\
\hline Donor African-American, \% & 25.0 & 28.3 & 0.223 \\
\hline Living donor, $\%$ & 16.3 & 16.2 & 0.989 \\
\hline KDPI, \% & $46.9 \pm 27.0$ & $50.3 \pm 26.8$ & 0.068 \\
\hline HLA mismatches, median (IQR) & $4(3-5)$ & $4(3-5)$ & 0.372 \\
\hline Current PRA, median (IQR), \% & $0(0-35)$ & $0(0-31)$ & 0.561 \\
\hline Current PRA > 20\% & 32.0 & 29.7 & 0.439 \\
\hline Cold ischemic time, $\mathrm{h}$, mean $\pm \mathrm{SD}$ & $17.7 \pm 10.7$ & $18.3 \pm 10.6$ & 0.410 \\
\hline \multicolumn{4}{|l|}{ Induction therapy, \% } \\
\hline IL2RA induction & 60.8 & 53.0 & 0.012 \\
\hline Cytolytic induction & 38.2 & 45.0 & 0.027 \\
\hline \multicolumn{4}{|l|}{ Maintenance immunosuppression, \% } \\
\hline Tacrolimus & 97.3 & 97.8 & 0.650 \\
\hline Cyclosporine & 0.4 & 0.3 & 0.882 \\
\hline Mycophenolate & 97.4 & 96.5 & 0.387 \\
\hline Azathioprine & 0.1 & 0.0 & 0.613 \\
\hline mTOR inhibitor & 0.6 & 3.2 & $<0.001$ \\
\hline Steroid withdrawal & 2.8 & 2.9 & 0.924 \\
\hline
\end{tabular}

KDPI, Kidney Donor Profile Index; HLA, human leukocyte antigen; mTOR, mammalian target of rapamycin

Delayed graft function was more common in patients with eGFR CV $<30 \%$.

eGFR first-year peak was similar in both groups; however, patients in the eGFR $C V \geq 30 \%$ group tended to reach their peak earlier and progress to their minimum eGFR more rapidly. Patients in the eGFR CV $\geq 30 \%$ group also had a significantly lower minimum and mean eGFRs after the peak, when compared to patients in the eGFR $\mathrm{CV}<30 \%$ group. The slope after the peak eGFR was also significantly lower in the high variability group at $-11.2 \pm$ 13.3 vs. $-1.8 \pm 8.8 \mathrm{~mL} / \mathrm{min} /$ year $(p<0.001)$.

Estimated death-censored graft survival, overall graft, and patient survival were significantly lower in the patients who had higher eGFR variability, even after accounting for confounding and other eGFR measures (peak and slope). Multivariable logistic regression (Table
3) demonstrated that patients with high eGFR variability were more likely to have high tacrolimus trough variability and evidence of acute rejection, while mean eGFR after peak and eGFR slope after peak were protective against observed eGFR variability. When graft loss was considered the outcome variable (Table 4), logistic regression revealed that deceased donor, presence of acute rejection, and eGFR CV $>30 \%$ were significant predictors of loss. Mean eGFR after peak and eGFR slope after peak provided protection against graft loss. For death-censored graft loss (Table 5), age, female gender, mean eGFR after peak, and eGFR slope after peak were protective, while tacrolimus concentration variability, presence of acute rejection, and eGFR CV $>30 \%$ were significant predictors of death-censored graft loss. Table 6 evaluates factors associated with patient death. Increased age and eGFR 
Table 2. Post-transplant clinical outcomes compared by eGFR variability

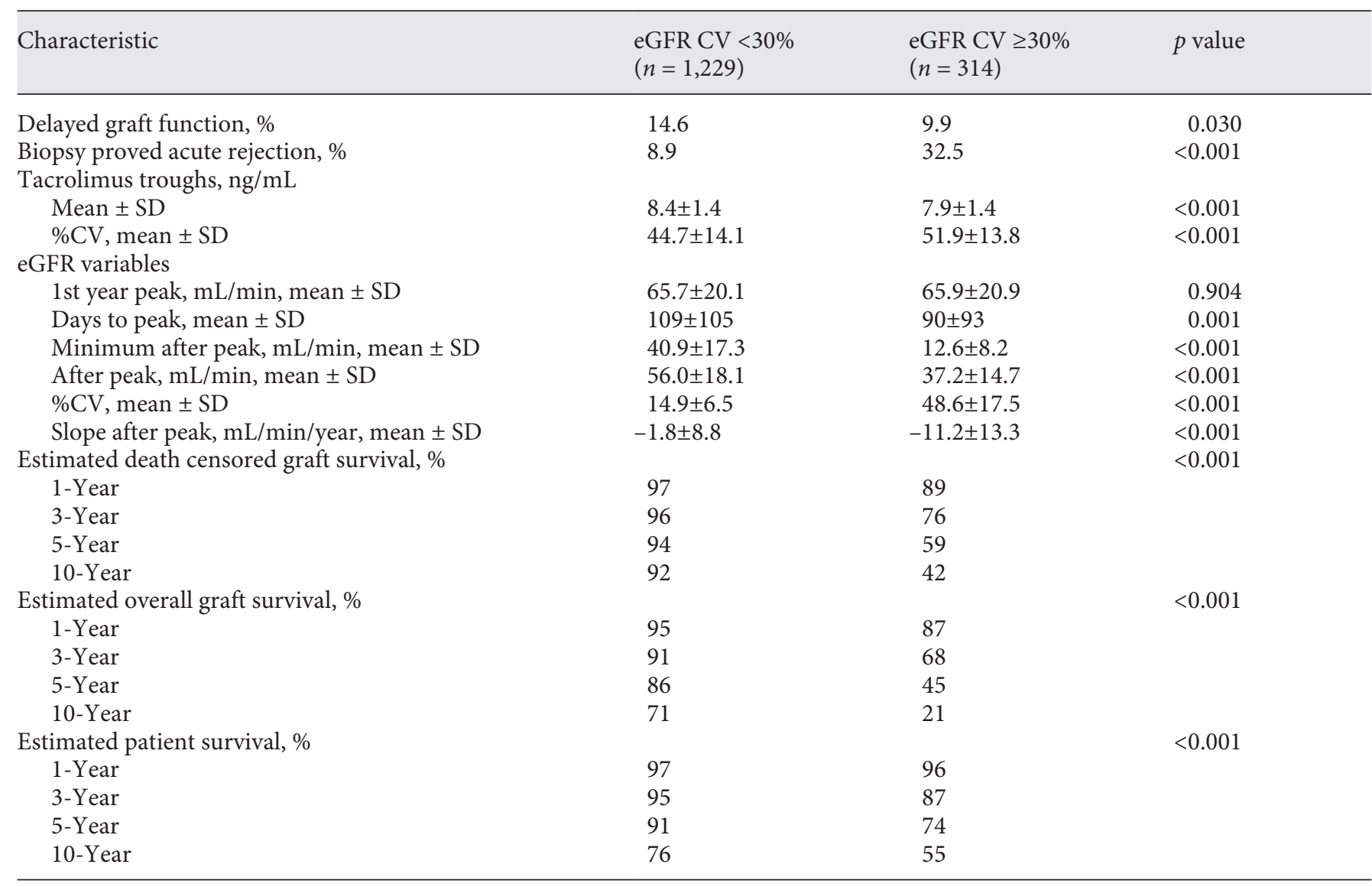

$\mathrm{CV}>30 \%$ were the only variables that were more likely to predict death, while mean eGFR after peak and eGFR slope after peak were significantly protective.

Cox regression analysis, adjusted for known variables that may influence graft loss, demonstrates that patients with eGFR variability of $>30 \%$ had significantly lower estimated death-censored and uncensored graft survival compared to those patients with $\leq 30 \%$ variability (Fig. 1, 2). Figure 3 also demonstrates a similar trend for adjusted estimated patient survival, although the comparison between those patients with $\leq 30 \%$ variability and $>30 \%$ variability did not reach statistical significance.

\section{Discussion}

Traditional surrogate markers of long-term kidney allograft outcomes, such as acute rejection, have become mostly obsolete in the current era of transplantation [16]. In the setting of modern immunosuppression and im-
Table 3. Variables significantly associated with high post-transplant, high eGFR variability

\begin{tabular}{lcrr}
\hline Variable & OR & $95 \%$ CI & $p$ value \\
\hline $\begin{array}{l}\text { Donor age, years } \\
\text { Cytolytic induction therapy }\end{array}$ & 0.98 & $0.96-0.99$ & 0.001 \\
Tacrolimus trough & & $0.97-2.07$ & 0.071 \\
$\quad$ variability (\%CV) & 1.02 & $1.01-1.04$ & $<0.001$ \\
Acute rejection & 1.95 & $1.22-3.11$ & 0.005 \\
1st year peak eGFR & 1.11 & $1.09-1.12$ & $<0.001$ \\
Days to peak eGFR & 0.99 & $0.99-1.00$ & 0.049 \\
Mean eGFR after peak & 0.84 & $0.82-0.86$ & $<0.001$ \\
eGFR slope after peak & 0.96 & $0.94-0.98$ & $<0.001$ \\
\hline
\end{tabular}

mune monitoring, bedside clinical measures, beyond serum creatinine, are needed to determine the "health" of the kidney allograft.

This study represents novel analysis of a large cohort of patients demonstrating that the slope of GFR and 
eGFR variability was identified as strong independent predictors of graft outcomes. Patients that demonstrated significant eGFR slope variability, defined as CV $\geq 30 \%$, were more likely to experience unfavorable longterm allograft outcomes. More importantly, the variability in the eGFR slope likely provides an additional level of detail to target patients for intervention. These data also introduce several eGFR variables including first-year eGFR peak, time to peak, minimum after peak, mean after peak, and slope after peak. Introduction of additional variables to describe patient's peak allograft performance and post-peak eGFR trajectory should allow for improved targeting of patients for intervention to mitigate risk of long-term allograft failure. In comparison to finite static measurements of eGFR or even slope of eGFR, eGFR variability demonstrates perhaps how events within a patient's post-transplant course can significantly impact their trajectory, such as acute kidney injury events.

Specifically, our data indicate that patients with the high eGFR variability were younger, female and more frequently had rejection despite receiving more cytolytic induction therapy. Limited studies of slope of eGFR and trajectory to graft failure or patient death are published. Therefore, we compare our findings to studies published in the end-stage renal disease population. Perkins et al. [17] reported on their analysis of a longitudinal electronic database in which they collated data from patients with stage III CKD and sought to evaluate eGFR variability in predicting progression to ESRD. Like our study, this was retrospective and included adult patients with at least 4 GFR readings and their study endpoint was time to eGFR $<15 \mathrm{~mL} / \mathrm{min} / 1.73 \mathrm{~m}^{2}$, dialysis, or transplant. In this study, patients were divided into 4 cohorts of eGFR variability. Interestingly, eGFR variability did not predict the primary outcomes even after being adjusted for known risk factors for CKD progression but may demonstrate trajectory to ESRD versus death. Authors suggest that eGFR variability examined at different points in the patient's clinical course and may aid in deciphering cardiovascular outcomes and as we suggest provide a surrogate measure for use in clinical practice [17]. Grams et al. [18] also evaluated progression to ESRD in 1,094 patients enrolled in a multicenter randomized controlled trial to evaluate the efficacy of antihypertensive medications and 2 different blood pressure control targets for a median of 8 years. Patients were divided into 5 groups and evaluated based on a predetermined baseline eGFR or risk for ESRD.
Table 4. Predictors of graft loss

\begin{tabular}{lccc}
\hline Variable & HR & $95 \%$ CI & $p$ value \\
\hline Age, years & 1.01 & $1.01-1.02$ & 0.002 \\
Gender, female & 0.69 & $0.55-0.88$ & 0.003 \\
Deceased donor & 2.14 & $1.43-3.19$ & $<0.001$ \\
Acute rejection & 1.79 & $1.36-2.36$ & $<0.001$ \\
Mean eGFR after peak & 0.97 & $0.96-0.97$ & $<0.001$ \\
eGFR slope after peak & 0.94 & $0.93-0.95$ & $<0.001$ \\
eGFR CV $>30 \%$ & 1.91 & $1.47-2.49$ & $<0.001$ \\
\hline
\end{tabular}

Table 5. Predictors of death-censored graft loss

\begin{tabular}{lccr}
\hline Variable & HR & $95 \%$ CI & $p$ value \\
\hline Age, years & 0.97 & $0.96-0.99$ & $<0.001$ \\
Gender, female & 0.45 & $0.32-0.64$ & $<0.001$ \\
HLA mismatch & 1.11 & $1.00-1.23$ & 0.058 \\
Deceased donor & 1.78 & $1.04-3.07$ & 0.036 \\
Acute rejection & 2.71 & $1.90-3.86$ & $<0.001$ \\
Tacrolimus trough & & & \\
$\quad$ variability (\%CV) & 1.01 & $1.01-1.02$ & 0.002 \\
Mean eGFR after peak & 0.94 & $0.93-0.95$ & $<0.001$ \\
eGFR slope after peak & 0.93 & $0.92-0.94$ & $<0.001$ \\
eGFR CV $>30 \%$ & 2.00 & $1.34-3.00$ & 0.001 \\
\hline
\end{tabular}

Table 6. Predictors of death

\begin{tabular}{lccr}
\hline Variable & HR & $95 \%$ CI & $p$ value \\
\hline Age, years & 1.05 & $1.04-1.06$ & $<0.001$ \\
African-American & 0.66 & $0.48-0.91$ & 0.012 \\
Years on dialysis & 1.05 & $0.99-1.11$ & 0.079 \\
Deceased donor & 1.50 & $0.91-2.47$ & 0.111 \\
Mean eGFR after peak & 0.99 & $0.98-0.99$ & 0.003 \\
eGFR slope after peak & 0.97 & $0.96-0.98$ & $<0.001$ \\
eGFR CV $>30 \%$ & 1.47 & $1.04-2.07$ & 0.028 \\
\hline
\end{tabular}

Interestingly, in multivariate analysis, patients who started with an eGFR of $30 \mathrm{~mL} / \mathrm{min} / 1.73 \mathrm{~m}^{2}$ were more likely to progress to ESRD if they were younger [18]. Uehara et al. [19] also performed a post hoc analysis of a pharmacologic intervention trial to determine if patients with advanced kidney disease who had higher eGFR variability would hasten their progression to ESRD requiring transplant or dialysis. A total of 237 patients were included in their final analysis and contrary to our findings, patients with high eGFR variabil- 
Fig. 1. Adjusted* estimated death-censored graft survival compared by eGFR variability. * Adjusted age, sex, race, ESRD diagnosis, HLA mismatches, PRA, cold ischemic time, years on dialysis, donor age, donor sex, donor race, deceased donor, cytolytic induction, tacrolimus trough mean, tacrolimus variability $(\% \mathrm{CV})$, delayed graft function, acute rejection, peak eGFR, mean eGFR, days to peak eGFR, and slope of eGFR after peak.

Fig. 2. Adjusted* estimated graft survival compared by eGFR variability. * Adjusted age, sex, race, ESRD diagnosis, HLA mismatches, PRA, cold ischemic time, years on dialysis, donor age, donor sex, donor race, deceased donor, cytolytic induction, tacrolimus trough mean, tacrolimus variability $(\% \mathrm{CV})$, delayed graft function, acute rejection, peak eGFR, mean eGFR, days to peak eGFR, and slope of eGFR after peak.

Fig. 3. Adjusted* estimated patient survival compared by eGFR variability. * Adjusted age, sex, race, ESRD diagnosis, HLA mismatches, PRA, cold ischemic time, years on dialysis, donor age, donor sex, donor race, deceased donor, cytolytic induction, tacrolimus trough mean, tacrolimus variability $(\% \mathrm{CV})$, delayed graft function, acute rejection, peak eGFR, mean eGFR, days to peak eGFR, and slope of eGFR after peak.

Renal Function and Graft Loss in Kidney Transplantation
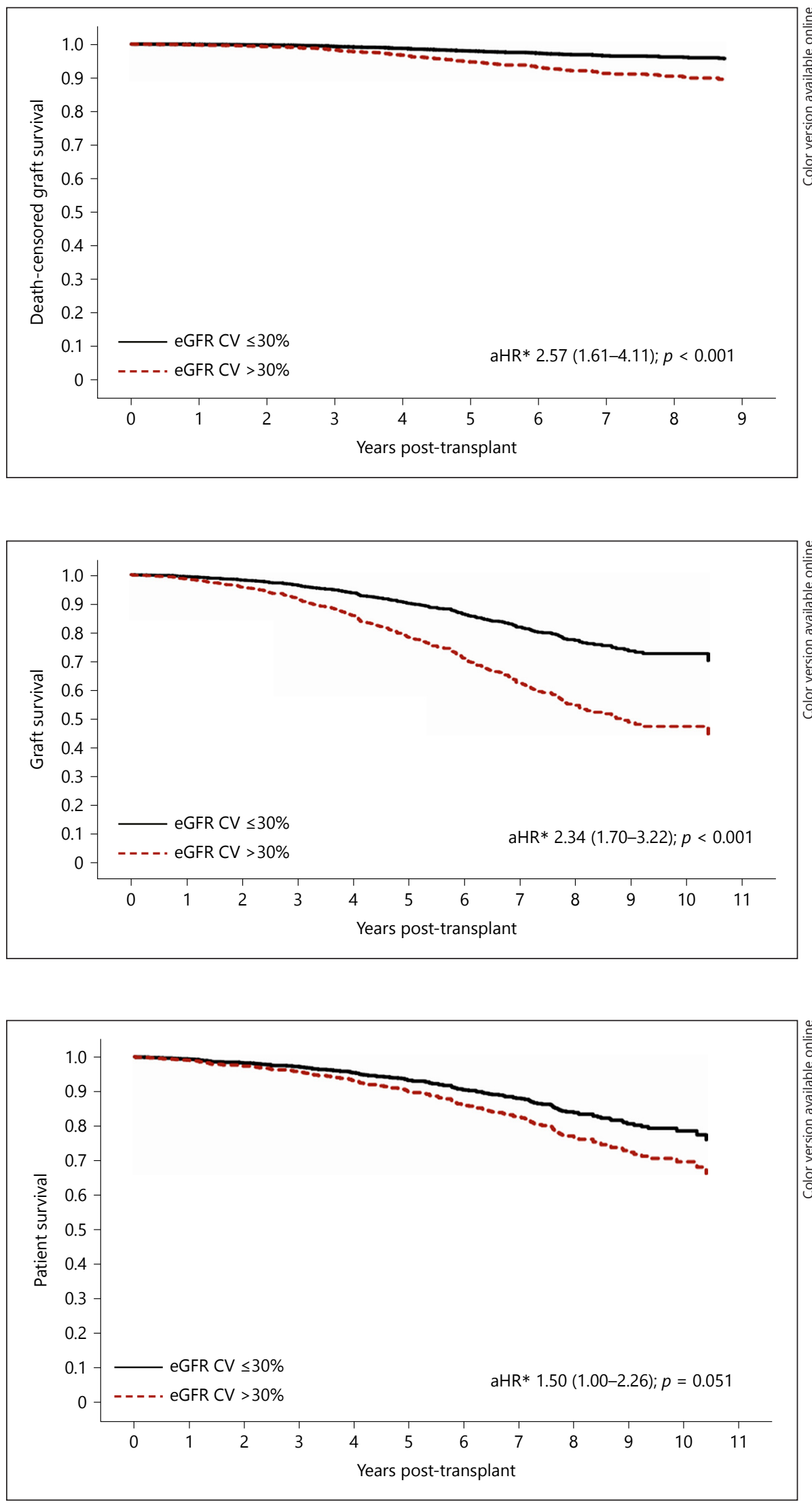

Am J Nephrol 2018;47:191-199 
ity tended to be older (median 68 years vs. 62.5 years in low eGFR variability group, $p<0.001$ ) and male (94 vs. $76 \%$ in low eGFR variability group). However, similar to our findings, patients with high eGFR variability were 2.1 times more likely to experience progression to ESRD, requiring transplant or dialysis [19]. Authors indicate that visit-to-visit variability in eGFR should be evaluated on a larger scale and that eGFR slope and eGFR variability may be useful in predicting outcomes [19].

High tacrolimus level variability was also seen in conjunction with high eGFR variability in several of our graft outcome models as predictors of poorer outcomes. This variable, although not the crux of our analysis, may also indicate that laboratory variability measures can provide a surrogate marker for identification of potential nonadherence. Huang et al. [20] recently published a study detailing the link between higher variability in tacrolimus trough levels and risk for acute rejection in kidney transplant recipients. Their focus was on kidney transplant recipients who experienced acute rejection between 2012 and 2014 and were compared to patients without rejection. Patients who experienced acute rejection had significantly higher variability in their tacrolimus trough levels against those that did not experience rejection $(39 \pm 15.6 \%$ vs. $12.1 \pm 7.9 \%$ in the control group, $p<0.001$ ) [20]. Authors suggest, as we have, that this may be a marker of nonadherence but also acknowledge, as we do know that other factors such as genetic metabolic differences, drug interactions or formulation may also contribute to the variability etiology [20]. eGFR variability is a strong predictor of allograft outcomes in our kidney transplant recipients. Additional studies are needed to determine the true impact of eGFR variability as an outcome measure used to target patients for earlier intervention.

Our study is not without limitations. This was a retrospective review of a large, single-center cohort. Calculations of slope of eGFR may have been based on a different number of laboratory values depending on the patient's exposure to the health care system. Those patients who had higher variability may have required more laboratory monitoring making the variability more pronounced as we know that episodes such as dehydration or infection may produce variability and additional monitoring. Also those patients with acute rejection episodes likely had more laboratory values drawn around their rejection episode, which may have impacted the variability. However, we aimed to describe the trajectory of eGFR using additional variables to describe the slope, such as first-year
eGFR peak, time to peak, minimum after peak, mean after peak, and slope after peak. Also, this study did span over 10 years. Programmatic changes in immunosuppression may have impacted our results. We primarily used IL2RA induction for patients with a panel reactive antibody $<80 \%$ and cold ischemia times $<24 \mathrm{~h}$. T-cell depleting induction was reserved for highly sensitized patients. Our center also endorses of having achieved a level of at least $8 \mathrm{ng} / \mathrm{mL}$ for tacrolimus by $48 \mathrm{~h}$ with IL2RA induction and by the completion of T-cell depleting induction post transplant. We also did increase the rapidity of our corticosteroid taper from 2005 to 2015. By 2015, corticosteroids were reduced to $5 \mathrm{mg}$ daily by day 45 post transplant.

In conclusion, the slope of eGFR, used in concert with eGFR variability, is a strong predictor of long-term allograft outcomes. The development of electronic medical record signaling tools to allow clinicians access the patient's slope of eGFR when visits and labs are less frequent and target patients for additional follow-up eGFR variability may be extremely beneficial to aid quick assessment of post-transplant risk for long-term allograft loss and allow for tailored interventions. Additional analyses are needed to determine if there is a causal link between eGFR variability and allograft loss, while also assessing for potential interventions, which may mitigate this risk.

\section{Disclosure Statement}

The authors of this manuscript have no conflicts of interest to disclose related to this study. The contents of this paper have not been published previously.

\section{Funding}

This study was supported by NIH NIDDK K23.

\section{Author Contributions}

N.A.P.: Data analysis/interpretations, drafting article. V.R.: Concept/design, critical revision of article, approval of article. V.R.: Concept/design, critical revision of article, approval of article. P.D.M.: Critical revision of article, approval of article. Z.S.: Critical revision of article, approval of article. D.A.D.: Critical revision of article, approval of article. T.A.M.: Critical revision of article, approval of article. D.J.T.: Data analysis/interpretations, concept/design, critical revision of article, approval of article. 


\section{References}

1 Pippias M, Kramer A, Noordzij M, Afentakis $\mathrm{N}$, Alonso de la Torre R, Ambuhl PM, Aparicio Madre MI, Arribas Monzon F, Asberg A, Bonthuis M, Bouzas Caamano E, Bubic I, Caskey FJ, Castro de la Nuez P, Cernevskis H, de Los Angeles Garcia Bazaga M, des Grottes JM, Fernandez Gonzalez R, Ferrer-Alamar M, Finne P, Garneata L, Golan E, Heaf JG, Hemmelder MH, Idrizi A, Ioannou K, Jarraya F, Kantaria N, Kolesnyk M, Kramar R, Lassalle M, Lezaic VV, Lopot F, Macario F, Magaz A, Martin de Francisco AL, Martin Escobar E, Martinez Castelao A, Metcalfe W, Moreno Alia I, Nordio M, OtsRosenberg M, Palsson R, Ratkovic M, Resic H, Rutkowski B, Santiuste de Pablos C, Seyahi N, Fernanda Slon Roblero M, Spustova V, Stas KJF, Stendahl ME, Stojceva-Taneva $\mathrm{O}$, Vazelov E, Ziginskiene $\mathrm{E}$, Massy Z, Jager KJ, Stel VS: The European renal association - European dialysis and transplant association registry annual report 2014: a summary. Clin Kidney J 2017;10: 154-169.

2 Keith DS, Vranic G, Nishio-Lucar A: Graft function and intermediate-term outcomes of kidney transplants improved in the last decade: analysis of the United States kidney transplant database. Transplant Direct 2017; 3:e166.

3 Hart A, Smith JM, Skeans MA, Gustafson SK, Stewart DE, Cherikh WS, Wainright JL, Kucheryavaya A, Woodbury M, Snyder JJ, Kasiske BL, Israni AK: OPTN/SRTR 2015 annual data report: kidney. Am J Transplant 2017;17(suppl 1):21-116

4 Perez-Saez MJ, Montero N, Redondo-Pachon D, Crespo M, Pascual J: Strategies for an expanded use of kidneys from elderly donors. Transplantation 2017;101:727-745.

5 Gupta A, Francos G, Frank AM, Shah AP: KDPI score is a strong predictor of future graft function: moderate KDPI $(35-85)$ and high KDPI (>85) grafts yield similar graft function and survival. Clin Nephrol 2016;86: 175-182.
6 Querard AH, Foucher Y, Combescure C, Dantan E, Larmet D, Lorent M, Pouteau LM, Giral M, Gillaizeau F: Comparison of survival outcomes between expanded criteria donor and standard criteria donor kidney transplant recipients: a systematic review and meta-analysis. Transpl Int 2016;29:403-415.

7 Hart A, Gustafson SK, Skeans MA, Stock P, Stewart D, Kasiske BL, Israni AK: OPTN/ SRTR 2015 annual data report: early effects of the new kidney allocation system. Am J Transplant 2017;17(suppl 1):543-564.

8 Melanson TA, Hockenberry JM, Plantinga L, Basu M, Pastan S, Mohan S, Howard DH, Patzer RE: New kidney allocation system associated with increased rates of transplants among black and hispanic patients. Health Aff (Millwood) 2017;36:1078-1085.

9 Lenihan CR, O'Kelly P, Mohan P, Little D, Walshe JJ, Kieran NE, Conlon PJ: MDRD-estimated GFR at one year post-renal transplant is a predictor of long-term graft function. Ren Fail 2008;30:345-352.

10 Lam NN, Tonelli M, Lentine KL, Hemmelgarn B, Ye F, Wen K, Klarenbach S: Albuminuria and posttransplant chronic kidney disease stage predict transplant outcomes. Kidney Int 2017;92:470-478.

11 Hassanain M, Simoneau E, Doi SA, Hebert MJ, Metrakos P, Tchervenkov J: An improved classification of kidney function recovery using estimated glomerular filtration rate slope post-transplantation. Transplant Proc 2016; 48:1993-1998.

12 Wu J, Li H, Huang H, Wang R, Wang Y, He $\mathrm{Q}$, Chen J: Slope of changes in renal function in the first year post-transplantation and one-yr estimated glomerular filtration rate together predict long-term renal allograft survival. Clin Transplant 2010;24: 862-868.

13 Park JS, Oh IH, Lee CH, Kim GH, Kang CM: The rate of decline of glomerular filtration rate is a predictor of long-term graft outcome after kidney transplantation. Transplant Proc 2013;45:1438-1441.
14 Choi HY, Huh KH, Lee JG, Song MK, Kim MS, Kim YS, Kim BS: Variability of the estimated glomerular filtration rate in the first year after kidney transplantation is an independent risk factor for poor renal allograft outcomes: a retrospective cohort study. PLoS One 2016;11:e0168337.

15 Levey AS, Bosch JP, Lewis JB, Greene T, Rogers N, Roth D: A more accurate method to estimate glomerular filtration rate from serum creatinine: a new prediction equation. modification of diet in renal disease study group. Ann Intern Med 1999;130:461-470.

16 Tong A, Budde K, Gill J, Josephson MA, Marson L, Pruett TL, Reese PP, Rosenbloom D, Rostaing L, Warrens AN, Wong G, Craig JC, Crowe S, Harris T, Hemmelgarn B, Manns B, Tugwell P, Van Biesen W, Wheeler DC, Winkelmayer WC, Evangelidis N, Sautenet B, Howell M, Chapman JR: Standardized outcomes in nephrology-transplantation: a global initiative to develop a core outcome set for trials in kidney transplantation. Transplant Direct 2016;2:e79.

17 Perkins RM, Kirchner HL, Hartle JE, Bucaloiu ID: Estimated glomerular filtration rate variability and risk of end-stage renal disease among patients with Stage 3 chronic kidney disease. Clin Nephrol 2013;80:256-262.

18 Grams ME, Li L, Greene TH, Tin A, Sang Y, Kao WH, Lipkowitz MS, Wright JT, Chang AR, Astor BC, Appel LJ: Estimating time to ESRD using kidney failure risk equations: results from the African American study of kidney disease and hypertension (AASK). Am J Kidney Dis 2015;65:394-402.

19 Uehara K, Yasuda T, Shibagaki Y, Kimura K Estimated glomerular filtration rate variability independently predicts renal prognosis in advanced chronic kidney disease patients. Nephron 2015;130:256-262.

20 Huang CT, Shu KH, Ho HC, Wu MJ: Higher variability of tacrolimus trough level increases risk of acute rejection in kidney transplant recipients. Transplant Proc 2016;48:19781980. 